\title{
Servicestrategien und Service-Angebotsdifferenzierung von ERP-Dienstleistern im deutschsprachigen Raum
}

\author{
Adrian Rüedy (D) · Roman Hänggi
}

Eingegangen: 11. April 2021 / Angenommen: 25. August 2021 / Online publiziert: 15. September 2021

(C) Der/die Autor(en) 2021

Zusammenfassung Im Kontext der vorliegenden Forschungsarbeit wird der Beitrag externer Enterprise-Resource-Planning-Dienstleister (ERP) im deutschsprachigen Raum sowie eine Möglichkeit zur Service-Angebotsdifferenzierung untersucht. Hierbei steht der Schweizer Produktionsstandort der Maschinen-, Elektro- und Metall-Industrie (MEM-Industrie) im Fokus. Gegenstand der Studie ist die Frage nach dem Beitrag externer Dienstleister während der Phasen der ERP-Implementierung und dem anschließenden Betrieb. Das Ergebnis ergänzt die bisherige Literatur zu kritischen Erfolgsfaktoren, welche bisher überwiegend im Zusammenhang mit dem Erfolg von ERP-Implementierungen im anwenderorientierten Kontext von Unternehmen untersucht wurde. Basierend auf einer Umfrage mit 59 Unternehmen, ermöglicht die vorliegende Arbeit die erbrachten Beiträge der ERP-Dienstleister in Verbindung mit den wichtigsten Erfolgsfaktoren einer ERP-Implementierung zu beurteilen. Unternehmen, die ein ERP-System etablierter Anbieter (z. B. SAP) einsetzen, sehen in der Implementierung einen höheren Nutzen in der Zusammenarbeit mit einem externen Dienstleister als Unternehmen, die spezifische und wenig umfassende Systeme im Einsatz haben. Den größten Mehrwert durch den Beitrag externer ERP-Dienstleister sehen die Unternehmen in der Qualitätsverbesserung bei der Implementierung sowie in der erhöhten Stabilität der IT-Infrastruktur. Der Beitrag zur gesteigerten Qualitätsverbesserung durch eine konsequente Datendurchgängigkeit kann als Ausgangspunkt zur Service-Angebotsdifferenzierung der ERP-Dienstleister gesehen werden. ERP-Systeme mit der Funktion eines Integrations-Hubs tragen somit zur nachhaltigen Qualitätsverbesserung bei und ermöglichen dadurch diverse Service-Differenzierungen.

Adrian Rüedy $(\bowtie) \cdot$ Roman Hänggi

OST Ostschweizer Fachhochschule, Rapperswil, Schweiz

E-Mail: adrian.rueedy@ost.ch 
Schlüsselwörter ERP Implementierung · Integrierter Datenfluss ·

Digitalisierungen · Digitale Transformation · Kritische Erfolgsfaktoren · ERP

Dienstleister

\title{
Service-Strategies and Service-Offering-Differentiation of ERP service providers in German-speaking countries
}

\begin{abstract}
This study contributes to the research on external Enterprise Resource Planning (ERP) service providers in the German-speaking countries and identifies potentials for differentiation of their service offering. Specifically, it focuses on the Swiss mechanical and electrical engineering industry (MEM Industry) and analyzes the added value of external service providers for the ERP implementation as well as subsequent operations and maintenance of the ERP system. The results add to the existing research on critical success factors, which have been studied mainly in the context of ERP implementation success within the ERP adopting companies. Based on data from a survey with 59 companies, this paper allows assessing the contributions of ERP service providers with respect to the major critical success factors of an ERP implementation. Companies using an ERP solution from one of the main software vendors (e.g., SAP) perceive external service providers more beneficial than companies that use more customized and, therefore, less comprehensive systems. According to the participants of this study, the greatest benefit of external ERP service providers is the quality improvement during the implementation phase as well as the increased stability of the IT infrastructure. An increased quality improvement through enhanced data consistency leads to a differentiation of service offerings. Thus, ERP systems with the function of an integration hub enable sustainable quality improvement and service differentiation.
\end{abstract}

Keywords ERP implementation · Integrated data flow · Digitalization · Digital transformation $\cdot$ Critical success factors · ERP service provider

\section{Einführung}

Unternehmen erkennen zunehmend den Nutzen ihrer Unternehmensdaten (z.B. Stammdaten, Bewegungsdaten) und damit verbundene Möglichkeiten zur Effizienzsteigerung. Die zu Grunde liegenden Unternehmensprozesse sowie eine integrierte Datendurchgängigkeit können die Kosten und das Leistungsvermögen von Unternehmen positiv beeinflussen. Es kann allerdings nur dann eine effiziente Wertschöpfung entstehen, wenn alle Unternehmensfunktionen miteinander harmonieren und als Einheit funktionieren, z. B. mittels einem Enterprise-Resource-Planning-System (ERP-Systeme). Wir verstehen unter ERP-Systemen eine integrierte Softwarelösung in Verbindung mit einer zentralen Datenbank, die es einem Unternehmen ermöglicht, den Informations- und Datenfluss in Echtzeit zu verwalten (Fui-Hoon Nah et al. 2001; Laudon und Laudon 2018). Ein Unternehmen ist ein komplexes Gebilde, welches es im ERP abzubilden gilt. Dies stellt die meisten Unternehmen unabhängig von der Unternehmensgröße vor Herausforderungen, welche mittels der Unterstüt- 
zung externer ERP-Dienstleister bewältigt werden können. Die Implementierung eines ERP-Systems wird in der Regel mit einem umfassenden Projekt realisiert. Solche Projekte beinhalten den Entscheid für ein ERP-System, das Business-ProcessReengineering der Unternehmensprozesse, das Abbilden respektive Konfigurieren der Prozesse im ERP-System und die Wahl eines geeigneten ERP-Dienstleisters zur Unterstützung bei diesen Aufgaben. Das Vorgehen in der Implementierung soll zudem berücksichtigen, ob das ERP-System in seiner Basis unverändert oder für den unternehmensspezifischen Kontext in einer angepassten Version implementiert werden soll (Hustad et al. 2016).

Aus der Literatur geht hervor, dass erfolgreiche ERP-Implementierungsprojekte von den internen IT-Ressourcen und dem externen Dienstleister stark abhängen (Elragal und Haddara 2013). Die externen ERP-Dienstleister decken die bestehenden Wissenslücken im Umgang mit ERP-Systemen oder das Defizit an benötigten Ressourcen ab (Motiwalla und Thompson 2012). Unternehmen greifen hier somit auf die Kompetenz eines ERP-Dienstleisters zurück. Dies zeigt sich sowohl an der umfangreichen und zunehmenden Einbindung von externen Dienstleistern (Galal et al. 2012) als auch daran, dass die Literatur den Einsatz von ERP-Dienstleistern als einen kritischen Erfolgsfaktor (KEF) definiert (Ram et al. 2013; Alsulami et al. 2016; Lech 2016; Leyh und Muschick 2013; Leyh 2015, 2014). Eine erfolgreiche ERP-Implementierung bedingt die Berücksichtigung der am häufigsten genannten KEFs für ein Unternehmen (Ram et al. 2013; Tarhini et al. 2015). Dazu gehört auch der Beitrag externer Dienstleister, die ERP-Systeme bereits wiederholt implementiert haben (Xin und Choudhary 2019).

Bereits zahlreiche Studien identifizieren kritische Erfolgsfaktoren (KEFs) im Zusammenhang mit dem Erfolg einer ERP-Implementierung in einem anwenderorientierten Kontext (Nagpal et al. 2015; Alsulami et al. 2016; Lech 2016; Leyh und Muschick 2013; Leyh 2014; Ram et al. 2013). Diese Studien untersuchen hauptsächlich Auswirkungen für die anwendenden Unternehmen, beleuchten dabei jedoch selten die Tätigkeit der ERP-Dienstleister selbst. Jæger et al. (2020) halten in ihrer Studie fest, dass es angesichts der Bedeutung von externen Dienstleistern für eine erfolgreiche ERP-Implementierung unerlässlich ist, diese Schlüsselkomponenten besser zu verstehen.

Diese Studie untersucht, inwieweit die für Unternehmen identifizierten KEFs für einen externen Berater relevant sind. Genauer ist die Frage nach dem Beitrag externer Dienstleister während der ERP-Implementierung sowie dem anschließenden Betrieb Gegenstand dieser Untersuchung. Dies führt zur Forschungsfrage, welche grundlegenden Differenzierungspotenziale und Unterschiede von ERP-Dienstleistern bei der Einführung von ERP-Systemen identifiziert werden können.

Die Ergebnisse beziehen sich auf die Servicestrategie von ERP-Dienstleistern im deutschsprachigen Raum und verweisen auf Möglichkeiten zur Service-Angebotsdifferenzierung. Hierbei steht der Schweizer Produktionsstandort der Maschinen-, Elektro- und Metall-Industrie (MEM-Industrie) im Fokus. 59 Unternehmen wurden befragt, ob die in der Forschung breit diskutierten KEFs für externe Dienstleister relevant sind und wie groß der geleistete Beitrag ist. 


\section{ERP-Integration im Zusammenhang mit der Industrie 4.0}

Der Nutzen der Integration von ERP-Systemen wird vermehrt mit dem integrierten Datenfluss über den ganzen Produktlebenszyklus im Unternehmen gesehen (Nyffenegger et al. 2018). Zusätzlich gewinnt die Einbindung von Produktionsmaschinen im Rahmen der ,intelligenten Fabrik' (vom engl. Smart Factory) an Bedeutung (Haddara und Elragal 2015; Gerbert et al. 2015). Eine Studie aus dem Jahr 2017 zeigt, dass über 50 Methoden und Referenzmodelle in der Literatur einen Bezug zur ERP-Implementierung herstellen (Taterh 2017). Es ist anzumerken, dass die Erfahrung bei der ERP-Implementierung branchenabhängig ist. Herausforderungen und Risiken bei der ERP-Implementierung sind folglich nach den branchenspezifischen Erfahrungen in der Praxis zu bewerten (Taterh 2017). Auf Grund dieser Unterschiede legt diese Arbeit den Fokus auf die MEM-Industrie. Der Schweizer Produktionsstandort der Maschinen-, Elektro- und Metall-Industrie sieht sich auf Grund des starken Schweizer Frankens und neuer technologischer Möglichkeiten mit verschiedenen Herausforderungen konfrontiert (Swissmem 2019b).

Um das Potenzial von ERP-Systemen in der Industrie 4.0 voll ausschöpfen zu können, benötigt es ein vertieftes Verständnis hinsichtlich der Unternehmensprozesse sowie des integrierten Datenflusses. Besonders für kleine und mittlere Unternehmen (KMUs) ergeben sich durch die Digitalisierung große Chancen zur Steigerung der Wettbewerbsfähigkeit am Produktionsstandort Schweiz (Finkler 2017; Cannellotto 2018). Die Realität zeigt, dass hohe Fehleinschätzungen hinsichtlich der geplanten Kosten bei der Implementierung von ERP-Systemen ein großes Problem darstellen (Haddara 2012; Panorama Consulting Group 2020). Nach einer Studie der Panorama Consulting Group (2020) erreichen nur etwas mehr als die Hälfte der Unternehmen ihre Budgetziele (62\%) sowie den erwarteten Nutzen (61\%) aller messbaren Ziele nach der Implementierung.

Im Betrieb verzeichnen Cloud-Lösungen gegenüber konventionellen On-Premise ERP-Systemen niedrigere Kosten, eine Skalierbarkeit des Systems, verbesserte Zugänglichkeiten, hohe Verfügbarkeit und eine einfachere Aktualisierung (Elmonem et al. 2016). Darüber hinaus erleichtert die Umstellung auf ein cloudbasiertes ERP den Fernzugriff auf das System (Muslmani et al. 2018). Aus der Studie der Panorama Consulting Group (2020) geht hervor, dass sich im Jahr 2020 mehr als die Hälfte der Unternehmen (63\%) für eine cloudbasierte Softwarelösung entschieden haben.

Mit der vierten industriellen Revolution erweitert sich das Aufgabenspektrum des ERP-Systems hin zu einem Integrations-Hub. Dabei werden Daten aus unterschiedlichen Quellen miteinander verknüpft und interpretiert (Finkler 2017; Meissner et al. 2017). Die Rolle des ERP-Systems als ein zentrales Datenbanksystem muss in Folge der steigenden Relevanz der Datendurchgängigkeit überdacht werden. Der Wandel eines ERP-Systems hin zu einem Integrations-Hub setzt mobile rollenbasierte Benutzerinteraktionen voraus (Bartodziej et al. 2017). Bei der Maschine-zu-Maschine (M2M)- und Maschine-zu-ERP-Kommunikation gibt es allerdings noch einige Herausforderungen, da keine einheitlichen Standards und Protokolle existieren (Haddara und Elragal 2015; Gerbert et al. 2015). 
Die Voraussetzung einer Echtzeit-Zweiwege-Kommunikation zwischen Maschinen, Prozessen und Produkten muss vom jeweiligen ERP-System unterstützt werden (Haddara und Elragal 2015). Darüber hinaus werden ERP-Systeme in Bezug auf die zunehmende vertikale Integration der Fertigungsebene als Teil des Manufacturing Execution System (MES) gefordert (Meissner et al. 2017). Ein MES zur Steigerung von Produktivität und Durchlaufzeit ermöglicht im Austausch mit dem ERP-System proaktives Handeln, wenn Qualitätserzeugnisse zeitnah und kosteneffizient ausgeliefert werden sollen. Cloudbasierte Lösungen und Internet of Things (IoT) dienen an dieser Stelle als erweiterte Datenquellen für das MES (Haddara und Elragal 2015). ERP-Systeme in Verbindung mit einem integrierten Datenfluss sind in der Lage, durch den Einsatz von künstlicher Intelligenz schnellere und bessere Entscheidungen mit geringerer Fehlerwahrscheinlichkeit zu treffen. Das maschinelle Lernen als ein Teil der künstlichen Intelligenz ist einer der wichtigsten Faktoren, die ERPSysteme zukünftig beeinflussen werden (Brierley 2019).

Die aufgeführten Themen sollen aufzeigen, mit welcher Komplexität Unternehmen in Folge einer ERP-Implementierung sowie der zukünftigen Ausrichtung des Systems konfrontiert werden. Jæger et al. (2020) halten in ihrer Studie fest, dass externe Dienstleister entscheidend für den Erfolg bei der ERP-Implementierung sind. Aus Sicht der anwendenden Unternehmen wird beispielsweise der Wissenstransfers als ein Risikofaktor wahrgenommen, wenn es um die Beziehung zum externen Dienstleister geht (Jæger et al. 2020).

\section{Beitrag externer Dienstleister bei der ERP-Implementierung}

Einleitend in den Zielen dieser Studie wurde bereits erwähnt, dass es nur wenig Literatur zu KEFs bezüglich des geleisteten Beitrags von ERP-Dienstleistern gibt. Aufgrund der kritischen Rolle externer Dienstleister ist es notwendig, einen tieferen Einblick in die Dienstleistungsperspektive zu erhalten. Die komplexeren Fragen der Beziehungen zwischen dem externen Dienstleister und den Kunden-Unternehmen wurde kaum bearbeitet (Chang et al. 2013). Diese Arbeit trägt dazu bei, die Relevanz externer Dienstleister aus der Perspektive der Kunden-Unternehmen besser zu verstehen.

Jæger et al. (2020) halten fest, dass sich die KEFs für ERP-Dienstleister mit jenen der Unternehmen überschneiden - sie haben jedoch eine etwas andere Bedeutung für den externen Dienstleister. Daher werden zur Bewertung die allgemeingültigen Erfolgsfaktoren aus der Literatur herangezogen. Veranschaulicht wird dies in den nachfolgenden Abb. 1 und 2. Die KEF-Forschung für ERP-Implementierungen bedarf wegen sich verändernder Kundenanforderungen und technologischer Möglichkeiten einer ständigen Neubeurteilung (Leyh 2015). Eine von Leyh (2015) durchgeführte Studie zeigt ein Ranking der kritischen Erfolgsfaktoren auf Basis ihrer Erscheinungshäufigkeit in den untersuchten Publikationen. Es zeigt sich, dass nur wenige KEF den technologischen Faktoren zuzuordnen sind. Die Mehrheit gehört zu den organisatorischen Faktoren mit einer strategischen Ausrichtung (Leyh 2015).

Die Erfahrung bei der ERP-Implementierung variiert je nach Branche und Größe der Unternehmung. Dies ist mit ein Grund dafür, dass die Literatur unterschiedliche 


\begin{tabular}{|c|c|c|c|c|c|}
\hline Ranking & Kritische Erfolgsfaktoren (KEF) & 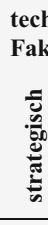 & gische & 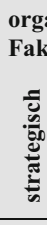 & 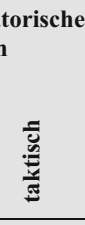 \\
\hline 01 & Projektmanagement & $\square$ & $\square$ & $\square$ & $凶$ \\
\hline 02 & Changemanagement & $\square$ & $\square$ & 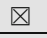 & $\square$ \\
\hline 03 & Anwenderschulungen & $\square$ & $\square$ & $\square$ & 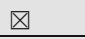 \\
\hline 04 & Unterstützung durch die Geschäftsführung & $\square$ & $\square$ & $\otimes$ & $\square$ \\
\hline 05 & Kommunikation & $\square$ & $\square$ & $\square$ & $凶$ \\
\hline 06 & Vorhandene interne Ressourcen & $\square$ & $\square$ & $凶$ & $\square$ \\
\hline 07 & $\begin{array}{l}\text { Konfiguration des ERP-Systems (z. B. Einbindung in } \\
\text { bestehende Infrastruktur, Einbindung der bestehenden oder } \\
\text { neuen Geschäftsprozesse) }\end{array}$ & $凶$ & $\square$ & $\square$ & $\square$ \\
\hline 08 & Zusammensetzung des Projektteams & $\square$ & $\square$ & $\unrhd$ & $\square$ \\
\hline 09 & Systemtests/Testverfahren & $\square$ & 凶 & $\square$ & $\square$ \\
\hline 10 & Klare Zielstellungen und Zielvorgaben & $\square$ & $\square$ & $凶$ & $\square$ \\
\hline 16 & Externe ERP-Berater bzw. Dienstleister & $\square$ & $\square$ & $\square$ & 凶 \\
\hline 25 & ERP-Anbieter-Beziehung und -Support & $\square$ & $\square$ & $凶$ & $\square$ \\
\hline
\end{tabular}

Abb. 1 Die zehn wichtigsten Erfolgsfaktoren für eine erfolgreiche ERP-Implementierung in Großunternehmen ergänzt mit dem Ranking der ERP-Dienstleister, in Anlehnung an Leyh und Muschick 2013

Erfolgsfaktoren aufzeigt. Zudem kann der Umfang der ERP-Projekte einen starken Einfluss auf die kritischen Erfolgsfaktoren haben, die den Erfolg bei der Implementierung und die Verbesserung der Unternehmenseffizienz erzielen sollen (A $\breve{g} a$ ğlu et al. 2015). In einer Studie aus dem Jahr 2013 wurden insgesamt 31 Faktoren identifiziert, die den ERP-Erfolg nach der Implementierung beeinflussen können (Leyh und Muschick 2013). Abb. 1 zeigt die zehn wichtigsten Erfolgsfaktoren für Großunternehmen und Abb. 2 bildet die Sicht auf klein- und mittelständische Unternehmen ab. Ergänzend wird zwischen technologischen und organisatorischen Einflüssen unterschieden (Leyh 2015; Leyh und Muschick 2013; Leyh 2014), die weiter nach der strategischen oder taktischen Ausprägung unterteilt werden (Leyh 2014; Al-Mudimigh et al. 2001). Ebenfalls ersichtlich in beiden Tabellen ist das Ranking der ERPDienstleister in Abhängigkeit zur Unternehmensgröße. Diese sind in der Abb. 1 auf Position 16 und Abb. 2 auf Position 12 zu finden.

Zwei unabhängige Studien stellen fest, dass die kritischen Erfolgsfaktoren hinsichtlich der Relevanz für KMUs im Vergleich zu Großunternehmen unterschiedlich priorisiert werden (Leyh 2015; Krcmar 2015). Die technologischen Faktoren einer ERP-Implementierung und ihre Auswirkungen auf KMUs können mit einer höheren Priorität bewertet werden. Dennoch sollten kleinere Unternehmen bei der Einführung eines ERP-Systems sowohl die organisatorischen als auch die technologischen Faktoren berücksichtigen. Die von Leyh (2015) durchgeführte Studie zeigt, dass die technologischen Faktoren - Systemtests/Testverfahren, Konfiguration des ERPSystems und Anpassungsmöglichkeiten des ERP-Systems - zu den fünf wichtigsten Erfolgsfaktoren für klein- und mittelständischen Unternehmen zählen (Leyh 2015). 


\begin{tabular}{|c|c|c|c|c|c|}
\hline Ranking & Kritische Erfolgsfaktoren (KEF) & 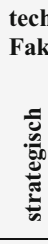 & 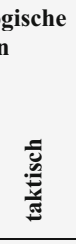 & 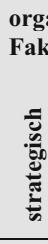 & torische \\
\hline 01 & Systemtests/Testverfahren & $\square$ & $凶$ & $\square$ & $\square$ \\
\hline 02 & $\begin{array}{l}\text { Passfähigkeit des ERP-Systems zur Organisationsstruktur } \\
\text { und branchenspezifische Besonderheiten }\end{array}$ & $凶$ & $\square$ & $\square$ & $\square$ \\
\hline 03 & Anwenderschulungen & $\square$ & $\square$ & $\square$ & $凶$ \\
\hline 04 & Klare Zielstellungen und Zielvorgaben & $\square$ & $\square$ & $\otimes$ & $\square$ \\
\hline 05 & $\begin{array}{l}\text { Konfiguration des ERP-Systems (z.B. Einbindung in } \\
\text { bestehende Infrastruktur, Einbindung der bestehenden } \\
\text { oder neuen Geschäftsprozesse) }\end{array}$ & $凶$ & $\square$ & $\square$ & $\square$ \\
\hline 06 & Zusammensetzung des Projektteams & $\square$ & $\square$ & $凶$ & $\square$ \\
\hline 07 & Unterstützung durch die Geschäftsführung & $\square$ & $\square$ & $\bigotimes$ & $\square$ \\
\hline 08 & Kommunikation & $\square$ & $\square$ & $\square$ & $凶$ \\
\hline 09 & Unternehmensstrategie/Unternehmensvision & $\square$ & $\square$ & $凶$ & $\square$ \\
\hline 10 & Projektleitung/Entscheidungskompetenzen & $\square$ & $\square$ & $\otimes$ & $\square$ \\
\hline 12 & Externe ERP-Berater bzw. -Dienstleister & $\square$ & $\square$ & $\square$ & $凶$ \\
\hline 14 & ERP-Anbieter-Beziehung und -Support & $\square$ & $\square$ & $凶$ & $\square$ \\
\hline
\end{tabular}

Abb. 2 Die zehn wichtigsten Erfolgsfaktoren für klein- und mittelständische Unternehmen ergänzt mit dem Ranking der ERP-Dienstleister, in Anlehnung an Leyh 2015, 2014

Ein Vergleich von Abb. 1 und 2 zeigt die unterschiedliche Bedeutung der kritischen Erfolgsfaktoren je nach Unternehmensgröße (Leyh 2014, 2015; Leyh und Muschick 2013). Sowohl die Beziehung als auch die Unterstützung beim Implementierungsprozess durch externe ERP-Dienstleister wird bei KMUs im Ranking auf Position 12 (vgl. Abb. 2) geführt (Leyh 2014). Bei Großunternehmen scheint dieser Erfolgsfaktor nahezu gleichbedeutend zu sein (Leyh und Muschick 2013).

Zudem konnte ein klarer Zusammenhang zwischen der Unternehmensgröße und dem Projektmanagement nachgewiesen werden. Während bei Großunternehmen ein eigenständiges ERP-Projektteam aufgestellt wird, geben ungefähr nur die Hälfte der klein- und mittelständischen Unternehmen an, ein Projektteam bei einer ERPImplementierung aufgestellt zu haben (Gärtner und Duller 2014).

Der Beitrag externer ERP-Dienstleister in der Implementierung - unabhängig von der Unternehmensgröße - ist als gleich groß zu erachten (Gärtner und Duller 2014). Die ERP-Dienstleister sind in den meisten Fällen auf ein spezifisches ERP-System eines Softwareunternehmens (nachfolgend auch ERP-Anbieter genannt) spezialisiert. Diese Abhängigkeit hat zur Folge, dass die Wahl des Dienstleisters eng mit den Produkteigenschaften des ERP-Anbieters verknüpft ist (Everdingen et al. 2000).

Am Beispiel des ERP-Softwareanbieters SAP kann aufgezeigt werden, dass in der Vergangenheit große Anstrengungen unternommen wurden, neue Dienstleistungspartner zu gewinnen. Im Zusammenhang mit dieser Entwicklung besteht ein Interessenskonflikt zwischen dem Wunsch seitens der ERP-Anbieter, mehr Partner zu zertifizieren, und dem Interesse der Kunden-Unternehmen, nur mit qualifizierten 
Partnern zu arbeiten. Dabei ist von besonderer Bedeutung, wie ERP-Anbieter ihre Partner zur Erbringung von Dienstleistungen anerkennen und zertifizieren (Elragal und Haddara 2013). Die Mehrheit der Schweizer Unternehmen ist mit ihrem ERPDienstleister und seinen Leistungen zufrieden, wobei die Zusammenarbeit zwischen den Unternehmen und den ERP-Dienstleistern mehrheitlich über fünf Jahre andauert (GIA Informatik AG 2018). Der Wunsch nach maßgeschneiderten Lösungen ist bei Schweizer Unternehmen groß. Dies betrifft vor allem die Integration von neuen ITSystemen und Funktionalitäten im Laufe des ERP-Lebenszyklus (GIA Informatik AG 2018).

Aus dem heutigen Stand der Forschung geht hervor, dass ERP-Dienstleister einen zentralen Beitrag zur erfolgreichen Implementierung von ERP-Systemen leisten. Dies zeigt sich sowohl an der zunehmenden Einbindung von externen Dienstleistern durch Unternehmen (Galal et al. 2012) als auch daran, dass die Literatur den Einsatz von ERP-Dienstleistern als einen kritischen Erfolgsfaktor definiert.

\section{Methodik}

Mit dem Ziel den Beitrag externer Dienstleister während der ERP-Implementierung und des Betriebs zu untersuchen, wurden Unternehmen unterschiedlicher Branchen und Größe innerhalb der Schweizer MEM-Industrie befragt. Für die Erhebung der notwendigen Daten eignet sich in diesem Fall die Forschungsmethode der Befragung. Bei der quantitativen Befragung handelt es sich um eine Primärforschung, bei welcher der Informationsbedarf durch die gezielte Erhebung der benötigten Daten ermittelt wird (Meffert 2015, S. 146f.). Die Umfrage umfasst Aspekte der kritischen Erfolgsfaktoren in der ERP-Implementierung und dem erzielten Unternehmensmehrwert im nachfolgenden Betrieb des Systems.

Die Grundgesamtheit der Umfrage besteht aus allen Unternehmen der Schweizer MEM-Industrie. Nach Aussage der Swissmem sind dies mehr als 31.000 Unternehmen (Swissmem 2019a).

Mit dem Ziel ein möglichst breites Teilnehmerfeld an Unternehmen für die Umfrage anzusprechen, wurden verschiedene Verbände und Netzwerke miteinbezogen. Unteranderem hat sich der führende Verband der Schweizer MEM-Industrie (Swissmem) sowie die Plattform Industrie 2025 mit dem Ziel, die digitale Transformation auf dem Werkplatz Schweiz voranzutreiben, aktiv an der Verbreitung der Umfrage beteiligt. Die Umfrage war zwei Monate lang über den Zeitraum vom 10.02.2020-10.04.2020 online über UmfrageOnline.ch zugänglich. Befragt wurde in sechs Themenblöcken mit insgesamt 28 Fragen:

- Unternehmensgröße und Branchenzugehörigkeit

- Verwendetes ERP-System und ERP-Anbieter

- Aktueller Stand im ERP-Lebenszyklus

- Beitrag des externen Beraters während der Implementierung des ERP-Systems

- Beitrag des externen Beraters im Betrieb/Unterhalt des ERP-Systems

- Technologien in Verbindung mit einem ERP-System 


\section{Ergebnisse}

Im Rahmen der Auswertung wurden nur die Antworten derjenigen Teilnehmer ausgewertet, welche die gesamte Umfrage ausgefüllt haben. Abb. 3 und 4 fassen die Forschungsergebnisse bezüglich des Beitrags externer Dienstleister zusammen. Auf einer 5er-Skala $(1=$ trifft nicht $\mathrm{zu} ; 5=$ trifft $\mathrm{zu})$ sind die Mittelwerte abgebildet. Abb. 3 veranschaulicht die Nützlichkeit der externen Dienstleister bezüglich der kritischen Erfolgsfaktoren in der ERP-Implementierung aus Sicht der Unternehmen. Ergänzend hält Abb. 4 den Beitrag der externen Dienstleister in Verbindung mit dem erzielten Nutzen eines ERP-Systems während des Betriebs fest.

Rund $70 \%$ der Unternehmen sind den kleinen und mittleren Unternehmen mit weniger als 250 Mitarbeitenden zuzuordnen. Lediglich $3 \%$ aller Unternehmen geben an, noch kein ERP-System zu verwenden. Unabhängig von der Unternehmensgröße haben rund $10 \%$ der Unternehmen aktuell eine Eigenentwicklung im Einsatz. Somit nutzen rund $87 \%$ der befragten Unternehmen ein Standard-ERP-System. $25 \%$ der befragten Unternehmen setzen bereits heute auf eine cloudbasierte ERP-Lösung.

Aus der Umfrage geht hervor, dass die ERP-Integration und -Implementierung die meistgenannten Gründe sind, weshalb ein Unternehmen auf die Erfahrung externer ERP-Dienstleister zurückgreift. Der nachhaltige Nutzen nach der Inbetriebnahme eines ERP-Systems ist abhängig vom Unterhalt der ERP-Landschaft. Aus Sicht der Dienstleister sind ERP-Wartung und -Support entscheidend, um die Kundenzufriedenheit und damit eine erfolgreiche ERP-Implementierung zu erzielen (Jæger et al. 2020). Law et al. (2010) untermauern die Bedeutung von Wartung und Support, indem diese Komponente zusammen mit dem Implementierungsergebnis entscheidend für den Gesamterfolg des ERP ist.

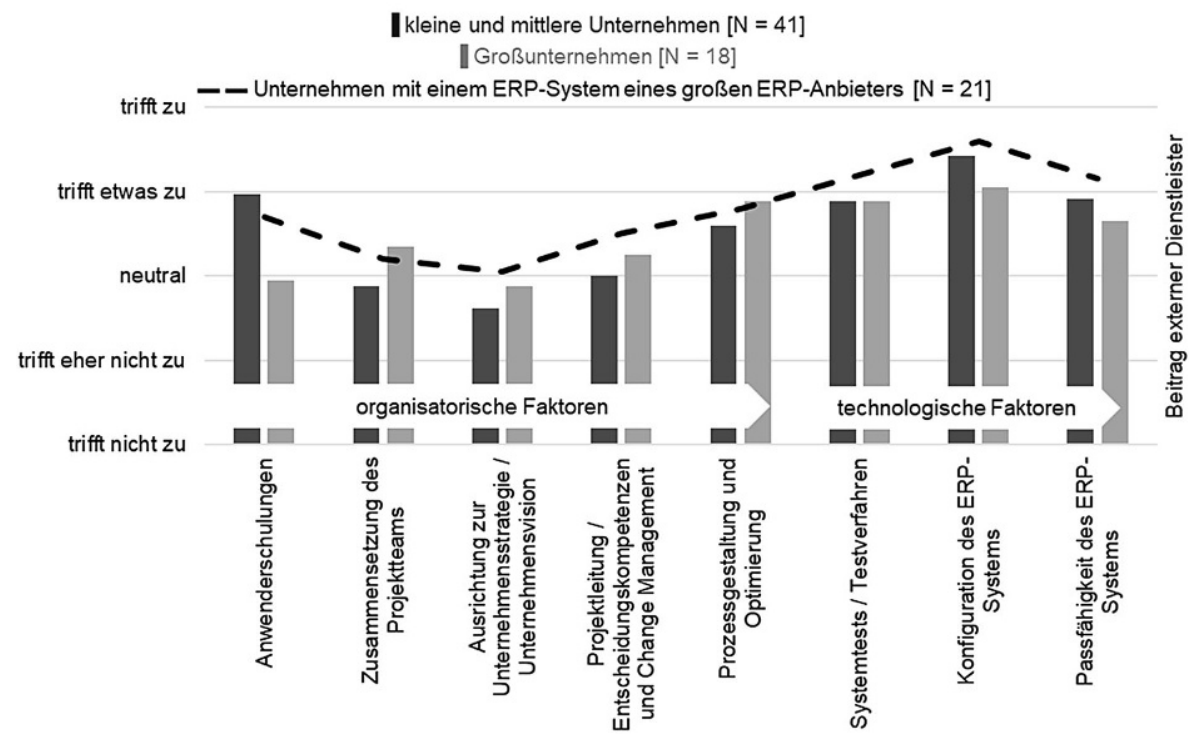

Abb. 3 Beitrag externer Dienstleister während der ERP-Implementierung 


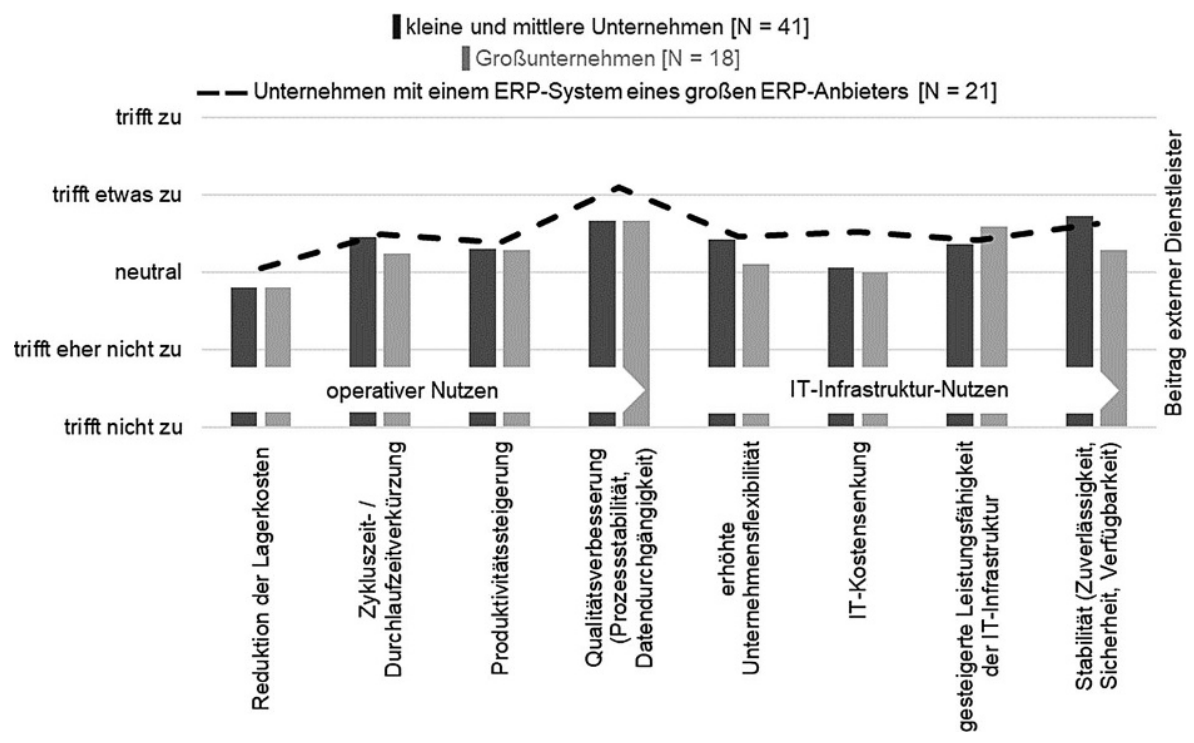

Abb. 4 Beitrag externer Dienstleister während des anschließenden Betriebs eines ERP-Systems

Das Gesamtbild der Umfrage hinsichtlich der ERP-Implementierung zeigt, dass der Beitrag der ERP-Dienstleister bezüglich der technologischen Faktoren am stärksten gewichtet wird. Die in die Umfrage einbezogenen technologischen Faktoren umfassen die Systemtests sowie die Konfiguration und die Passfähigkeit des ERPSystems. Alle übrigen Punkte sind den organisatorischen Faktoren zuzuordnen. In den meisten Fällen zeigen die organisatorischen Faktoren eine eher neutrale Gesamtwirkung auf den Nutzen des ERP-Dienstleisters für die Implementierung. Ein erhöhter Nutzen für die Unternehmen stellen die Anwenderschulung sowie die Prozessgestaltung und -optimierung dar. Die Schulung und das Training der ERP-Anwender beziehen sich auf die kontinuierliche Vermittlung von sowohl implizitem als auch explizitem Wissen. Infolge des Wissenstransfers über die Logik, das Konzept, die Prozesse und Funktionen des ERP-Systems kann über die Zeit die Effizienz im Umgang mit dem System verbessert werden (Ram et al. 2013). Mit Bezug auf den Beitrag externer Dienstleister im ERP-Betrieb kann festgestellt werden, dass keiner der aufgeführten Aspekte sich deutlich von den weiteren zur Auswahl stehenden Vorteilen abhebt. Den größten Mehrwert durch den Beitrag der ERP-Dienstleister sehen die Unternehmen in der Qualitätsverbesserung sowie in der Stabilität der ITInfrastruktur.

\subsection{Kleine und mittlere Unternehmen im Vergleich zu Großunternehmen}

Die Literatur hält fest, dass KEF einer ERP-Implementierung in Abhängigkeit zur Unternehmensgröße unterschiedlich gewichtet werden (Leyh 2015; Krcmar 2015). Die technologischen Faktoren einer ERP-Implementierung werden bei Großunternehmen weniger stark gewichtet. Dieser Zusammenhang kann auch in Verbindung mit dem Beitrag externer Dienstleister aufgezeigt werden. Die Konfiguration sowie 
die Passfähigkeit des ERP-Systems als technologische Faktoren werden von Großunternehmen im Gegensatz zur Grundgesamtheit als weniger bedeutend erachtet. Dies gilt auch für die Anwenderschulungen, die wohl eher durch unternehmensinterne Ressourcen abgedeckt werden. Einen höheren Stellenwert für Großunternehmen haben die externen Dienstleister in den Punkten Prozessgestaltung und -optimierung sowie Zusammensetzung des Projektteams und der Projektleitung. Großunternehmen weisen im Gegensatz zu den KMU einen geringeren Beitrag externer Dienstleister bei der erhöhten Unternehmensflexibilität und der Stabilität der IT-Architektur auf.

\subsection{Beitrag externer Dienstleister bei Verwendung eines ERP-Systems eines großen ERP-Anbieters}

Es wird davon ausgegangen, dass große ERP-Anbieter, wie beispielsweise SAP oder Microsoft, gemessen an der Mitarbeiterzahl ein größeres Investitionsvolumen zur Entwicklung neuer ERP-Lösungen bereitstellen. Dies ermöglicht den ERP-Anbietern, die Systemlandschaften der Unternehmen durch ein branchenübergreifendes ERP-System als Integrations-Hub zu zentralisieren (Meissner et al. 2017). Der Annahme zufolge kann der Markt so von einem integrierten Datenfluss durch die ERPLösung profitieren. Entsprechend der Annahme werden alle ERP-Lösungen, die von den Unternehmen genannt wurden und deren Anbieter weltweit mehr als 3000 Mitarbeitende beschäftigt, in der Auswertung berücksichtigt. Dies gilt für $21 \mathrm{der}$ befragten Unternehmen, darunter $11 \mathrm{KMU}$ sowie 10 Großunternehmen. Es zeigt sich, dass diejenigen Unternehmen, die ein ERP-System der großen Anbieter im Einsatz haben, in nahezu allen Punkten der Implementierung einen höheren Nutzen in der Zusammenarbeit mit einem externen Dienstleister sehen. Eine mögliche Interpretation wäre, dass das fundierte Wissen über die System-Abhängigkeiten und die entsprechende Datenstruktur bei den großen Anbietern ein zentraler Bestandteil der Implementierung ist. Dieses Know-how wird von den Kunden-Unternehmen durch die Leistungserbringung externer Dienstleister in Anspruch genommen. Dasselbe Bild zeigt sich auch während des Betriebs des ERP-Systems und dem daraus resultierenden Nutzen für das Unternehmen. Hierbei können Dienstleister vor allem in den Bereichen Qualitätsverbesserung und IT-Kostensenkung einen größeren Mehrwert beisteuern.

\section{Diskussion und Fazit}

ERP-Systeme haben den Anspruch, mittels der Integration einzelner betrieblicher Funktionen eine gesteigerte Leistungsfähigkeit der sie anwendenden Unternehmen zu erzielen. Das Ziel dieser Arbeit war die Analyse des aktuellen Beitrags von ERPDienstleistern bei ERP-Implementierungen in der Schweizer MEM-Industrie. Dazu wurden im Rahmen einer Umfrage insgesamt 59 Schweizer Unternehmen aus diversen Branchen der Maschinen-, Elektro- und Metall-Industrie mit unterschiedlicher Größe aus verschiedenen Schweizer Kantonen befragt. Die Umfrage lieferte 
Ergebnisse hinsichtlich des erwarteten Beitrags durch den Implementierungspartner in Folge eines ERP-Evaluationsprozesses.

Rund $88 \%$ der Unternehmen geben an, den Beitrag externer Dienstleister während des ERP-Lebenszyklus in Anspruch zu nehmen. Hinzu kommt, dass die Relevanz der externen Dienstleister in Abhängigkeit zur Unternehmensgröße und den einbezogenen Kriterien unterschiedlich zu bewerten ist. Die erhobenen Daten führen zu der Vermutung, dass der Beitrag und die Verwendungshäufigkeit von externen ERPDienstleistern mit zunehmender Größe des jeweiligen ERP-Anbieters steigen.

Die Bewältigung einer ERP-Implementierung kann Probleme mit sich bringen, welche durch den Einsatz von ERP-Dienstleistern verringert oder gar vermieden werden können. Die Mehrheit der befragten Unternehmen ist der Meinung, dass die Vorteile durch den Beitrag externer Dienstleister hinsichtlich der Konfiguration und Passfähigkeit des ERP-Systems am größten zu bewerten sind. Nach Angaben der Unternehmen liefern ERP-Dienstleister einen nachhaltigen positiven Einfluss auf die Effizienz im Unternehmen. Dies manifestiert sich in der Qualitätsverbesserung, die beispielsweise durch die Datenzuverlässigkeit bis hin zur Datendurchgängigkeit beeinflusst wird. Zudem ist die Mehrheit der großen Unternehmen der Überzeugung, dass Algorithmen für das Lernen auf Basis der grundlegenden Informationen eines ERP-Systems eine nachhaltige Steigerung der Effizienz bewirken können. Somit kann geschlussfolgert werden, dass der erwartete Beitrag eines ERP-Dienstleisters individuell auf das jeweilige Unternehmen $\mathrm{zu}$ beziehen ist und dementsprechend nicht generalisiert werden sollte.

Die Forschungsergebnisse sind insofern kritisch zu betrachten, da die Stichprobe keinesfalls als repräsentativ für die Grundgesamtheit der Schweizer MEM-Industrie anzusehen ist. Der Umfang und dementsprechend die Zahl der realisierten Elemente sind zu gering, als dass sich Thesen verifizieren oder valide Zusammenhänge zwischen den Befragungsergebnissen herstellen ließen. Die Ergebnisse der vorliegenden Arbeit eignen sich vorwiegend als deskriptive Messgrößen sowie dazu, allgemeine Tendenzen bezüglich des Forschungsthemas aufzuzeigen und können in zukünftiger Forschung entsprechend genutzt werden.

Funding Open access funding provided by University of Applied Sciences Eastern Switzerland

Open Access Dieser Artikel wird unter der Creative Commons Namensnennung 4.0 International Lizenz veröffentlicht, welche die Nutzung, Vervielfältigung, Bearbeitung, Verbreitung und Wiedergabe in jeglichem Medium und Format erlaubt, sofern Sie den/die ursprünglichen Autor(en) und die Quelle ordnungsgemäß nennen, einen Link zur Creative Commons Lizenz beifügen und angeben, ob Änderungen vorgenommen wurden.

Die in diesem Artikel enthaltenen Bilder und sonstiges Drittmaterial unterliegen ebenfalls der genannten Creative Commons Lizenz, sofern sich aus der Abbildungslegende nichts anderes ergibt. Sofern das betreffende Material nicht unter der genannten Creative Commons Lizenz steht und die betreffende Handlung nicht nach gesetzlichen Vorschriften erlaubt ist, ist für die oben aufgeführten Weiterverwendungen des Materials die Einwilligung des jeweiligen Rechteinhabers einzuholen.

Weitere Details zur Lizenz entnehmen Sie bitte der Lizenzinformation auf http://creativecommons.org/ licenses/by/4.0/deed.de. 


\section{Literatur}

Al-Mudimigh A, Zairi M, Al-Mashari M (2001) ERP software implementation: an integrative framework. Eur J Inf Syst 10:216-226. https://doi.org/10.1057/palgrave.ejis.3000406

Alsulami M, Scheepers H, Rahim MM (2016) A comparison between organizational Stakeholders' and external consultants' perceptions on CSFs affecting ERP life cycle phases. In: Bui TX, Sprague RH (Hrsg) Proceedings of the 49th Annual Hawaii International Conference on System Sciences. 5-8 January 2016, Kauai, Hawaii. IEEE, Piscataway, S 4676-4685

Ağaoğlu M, Yurtkoru ES, Ekmekçi AK (2015) The effect of ERP implementation CSFs on business performance; an empirical study on users' perception. Procedia Soc Behav Sci 210:35-42. https://doi. org/10.1016/j.sbspro.2015.11.326

Bartodziej C, Hochmuth CA, Schwägler C (2017) Industry 4.0 - Herausforderungen für ERP-Systeme im digitalen Wandel; Industry 4.0 - Is your ERP system ready for the digital era? Deloitte. https://www2. deloitte.com/de/de/pages/technology/articles/erp-industrie-4-0.html. Zugegriffen: 10. Jan. 2020

Brierley E (2019) Artificial Intelligence In ERP Software. Panorama Consulting Solutions. https://www. panorama-consulting.com/how-artificial-intelligence-improves-erp-software/. Zugegriffen: 24 . Nov. 2019

Cannellotto L (2018) ERP-Markt Schweiz 2018; Die Schweizer ERP-Landschaft. Profondia AG. https:// www.profondia.com/de/newsletter/erp-markt-schweiz-2018/. Zugegriffen: 28. Dez. 2019

Chang JYT, Wang ETG, Jiang JJ, Klein G (2013) Controlling ERP consultants: client and provider practices. J Syst Softw 86:1453-1461. https://doi.org/10.1016/j.jss.2013.01.030

Elmonem MA, Nasr ES, Geith MH (2016) Benefits and challenges of cloud ERP systems; a systematic literature review. Future Comput Inform J 1:1-9. https://doi.org/10.1016/j.fcij.2017.03.003

Elragal A, Haddara M (2013) The impact of ERP partnership formation regulations on the failure of ERP implementations. Procedia Technol 9:527-535. https://doi.org/10.1016/j.protcy.2013.12.059

van Everdingen Y, van Hillegersberg J, Waarts E (2000) ERP adoption by European midsize companies; searching for ERP systems offering a perfect fit. Commun ACM 43:27-31. https://doi.org/10.1145/ 332051.332064

Finkler M (2017) Industrie 4.0 - Wertschöpfung durch ERP-Systeme. Vogel Communications Group. https://www.industry-of-things.de/industrie-40-wertschoepfung-durch-erp-systeme-a-667815/. Zugegriffen: 30. Dez. 2019

Fui-Hoon Nah F, Lee-Shang Lau J, Kuang J (2001) Critical factors for successful implementation of enterprise systems. Business Process Mgmt Journal 7:285-296. https://doi.org/10.1108/ 14637150110392782

Galal K, Richter A, Wendlandt V (2012) It consulting and Outsourcing firms: evolution, business models, and future prospects. In: Kipping M, Clark T (Hrsg) The Oxford handbook of management consulting. Oxford University Press, Oxford, S 117-139

Gärtner B, Duller C (2014) Erfolgsfaktoren einer ERP-System-Implementierung; Eine vergleichende Analyse von Gross- und Mittelunternehmen. In: Feldbauer-Durstmüller B, Hiebl MRW (Hrsg) Controlling in Forschung und Praxis. 25 Jahre spezielle Betriebswirtschaftslehre „Controlling“ an der JKU Linz. Linde, Wien, S 151-178

Gerbert P, Lorenz M, Rüßmann M, Waldner M (2015) Industry 4.0: the future of productivity and growth in manufacturing industries. Boston Consulting Group. https://www.bcg.com/publications/2015/ engineered_products_project_business_industry_4_future_productivity_growth_manufacturing industries.aspx. Zugegriffen: 10. Jan. 2020

GIA Informatik AG (2018) Schweizer Unternehmen legen punkto Digitalisierung zu, starten aber noch nicht durch; Studie Digitalisierung. https://www.organisator.ch/schweizer-unternehmen-legenpunkto-digitalisierung-zu-starten-aber-noch-nicht-durch/. Zugegriffen: 22. Aug. 2021

Haddara M (2012) Exploring ERP adoption cost factors. Comput Technol Appl 3:250-261. https://doi.org/ $10.17265 / 1934-7332 / 2012.03 .007$

Haddara M, Elragal A (2015) The readiness of ERP systems for the factory of the future. Procedia Comput Sci 64:721-728. https://doi.org/10.1016/j.procs.2015.08.598

Hustad E, Haddara M, Kalvenes B (2016) ERP and organizational misfits: an ERP customization journey. Procedia Comput Sci 100:429-439. https://doi.org/10.1016/j.procs.2016.09.179

Jæger B, Bruckenberger SA, Mishra A (2020) Critical success factors for ERP consultancies; A case study. Scand J Inf Syst 32:169-202 
Krcmar H (2015) Kritische Erfolgsfaktoren und Herausforderungen von Software-as-a-Service basierten Enterprise Resource Planning Einführungsprojekten; Eine explorative Studie am Beispiel von SAP Business ByDesign

Laudon KC, Laudon JP (2018) Management information systems; managing the digital firm. Pearson, New York

Law CCH, Chen CC, Wu BJP (2010) Managing the full ERP life-cycle: considerations of maintenance and support requirements and IT governance practice as integral elements of the formula for successful ERP adoption. Comput Ind 61:297-308. https://doi.org/10.1016/j.compind.2009.10.004

Lech P (2016) Causes and remedies for the dominant risk factors in Enterprise system implementation projects: the consultants' perspective. SpringerPlus 5:238. https://doi.org/10.1186/s40064-016-18629

Leyh C (2014) Critical success factors for ERP projects in small and medium-sized enterprises - the perspective of selected German SMEs. In: Proceedings of the 2014 Federated Conference on Computer Science and Information Systems. IEEE, S 1181-1190

Leyh C (2015) Einflussfaktoren auf ERP-Projekte - Ergebnisse einer Interviewstudie ausgewählter KMU. ERP 1:45-47

Leyh C, Muschick P (2013) Critical success factors for ERP system upgrades - the case of a German large-scale enterprise Hyperconnected world: anything, anywhere, anytime. In: Proceedings of the 19th Americas Conference on Information Systems, (AMCIS-2013) ; Chicago, Illinois, USA, 15-17 August 2013. Curran, Red Hook

Meffert H (2015) Marketing; Grundlagen marktorientierter Unternehmensführung; Konzepte - Instrumente - Praxisbeispiele. Springer Gabler, Wiesbaden

Meissner J, Reschke J, Sontow K (2017) ERP-Lösungen 2017; Enterprise Resource Planning im Kontext von Industrie 4.0. Trovarit AG - the IT-Matchmaker. https://www.trovarit.com/wp-content/uploads/ erp-kontext-industrie-40.pdf. Zugegriffen: 22 Aug 2021

Motiwalla L, Thompson J (2012) Enterprise systems for management. Pearson, Boston

Muslmani BK, Kazakzeh S, Ayoubi E, Aljawarneh S (2018) Reducing integration complexity of cloudbased ERP systems. In: DATA '18: International Conference on Data Science, E-learning and Information Systems, S 1-6 https://doi.org/10.1145/3279996.3280033

Nagpal S, Khatri SK, Kumar A (2015) Comparative study of ERP implementation strategies. In: Long Island Systems, Applications and Technology. IEEE, S 1-9

Nyffenegger F, Hänggi R, Reisch A (2018) A reference model for PLM in the area of digitization. In: Chiabert P, Bouras A, Noël F, Ríos J (Hrsg) Product Lifecycle Management to Support Industry 4.0. 15th IFIP WG 5.1 International Conference, PLM 2018, Turin, Italy, July 2-4, 2018, Proceedings. Springer, Cham, S 358-366

Panorama Consulting Group (2020) The 2020 ERP report. https:/www.panorama-consulting.com/ resource-center/2020-erp-report/. Zugegriffen: 22 Aug 2021

Ram J, Corkindale D, Wu M (2013) Examining the role of system quality in ERP projects. Ind Manag Data Syst 113:350-366

Swissmem (2019a) Maschinen-, Elektro- und Metallindustrie (MEM). https://www.s-ge.com/de/maschinenelektro-und-metallindustrie. Zugegriffen: 10. März 2020

Swissmem (2019b) MEM-Industrie: Befürchtungen bestätigen sich; Der Abwärtstrend in der Schweizer Maschinen-, Elektro- und Metall-Industrie (MEM-Industrie) setzte sich im dritten Quartal 2019 unvermindert fort. https://www.swissmem.ch/de/aktuelles/detailansicht/news/mem-industriebefuerchtungen-bestaetigen-sich.html. Zugegriffen: 10. Jan. 2020

Tarhini A, Ammar H, Tarhini T, Masa'deh R (2015) Analysis of the critical success factors for enterprise resource planning implementation from stakeholders' perspective: a systematic review. IBR. https:// doi.org/10.5539/ibr.v8n4p25

Taterh S (2017) A study and analysis of various existing implementation framework related to enterprise resource planning. eecjournal 2:22-27. https://doi.org/10.24001/eec.2.3.4

Xin M, Choudhary V (2019) IT investment under competition: the role of implementation failure. Manage Sci 65:1909-1925. https://doi.org/10.1287/mnsc.2017.3005 